\title{
Optimal mechanism design for the private supply of a public good
}

Citation for published version (APA):

Csapó, G., \& Müller, R. J. (2012). Optimal mechanism design for the private supply of a public good. METEOR, Maastricht University School of Business and Economics. METEOR Research Memorandum No. 037 https://doi.org/10.26481/umamet.2012037

Document status and date:

Published: 01/01/2012

DOI:

10.26481/umamet.2012037

Document Version:

Publisher's PDF, also known as Version of record

\section{Please check the document version of this publication:}

- A submitted manuscript is the version of the article upon submission and before peer-review. There can be important differences between the submitted version and the official published version of record.

People interested in the research are advised to contact the author for the final version of the publication, or visit the DOI to the publisher's website.

- The final author version and the galley proof are versions of the publication after peer review.

- The final published version features the final layout of the paper including the volume, issue and page numbers.

Link to publication

\footnotetext{
General rights rights.

- You may freely distribute the URL identifying the publication in the public portal. please follow below link for the End User Agreement:

www.umlib.nl/taverne-license

Take down policy

If you believe that this document breaches copyright please contact us at:

repository@maastrichtuniversity.nl

providing details and we will investigate your claim.
}

Copyright and moral rights for the publications made accessible in the public portal are retained by the authors and/or other copyright owners and it is a condition of accessing publications that users recognise and abide by the legal requirements associated with these

- Users may download and print one copy of any publication from the public portal for the purpose of private study or research.

- You may not further distribute the material or use it for any profit-making activity or commercial gain

If the publication is distributed under the terms of Article $25 \mathrm{fa}$ of the Dutch Copyright Act, indicated by the "Taverne" license above, 


\section{Maastricht University}

Gergely Csapó, Rudolf Müller

Optimal mechanism design for the private supply of a public good

RM/12/037

\section{METEOR}

Maastricht University School of Business and Economics

Masstricht Research School of Economics

of Technology and Organization

PO. Box 616

NL-6200 MD Mastricht

The Netherlands 


\title{
Optimal mechanism design for the private supply of a public good
}

\author{
Gergely Csapó \\ Department of Quantitative Economics \\ Maastricht University \\ g.csapo@maastrichtuniversity.nl
}

\author{
Rudolf Müller * \\ Department of Quantitative Economics \\ Maastricht University \\ r.muller@maastrichtuniversity.nl
}

June 28, 2012

\begin{abstract}
We study the problem of finding the profit-maximizing mechanism for a monopolistic provider of a single, non-excludable public good. This problem has been well studied for the case when agents' types are independently distributed, but the literature is almost silent about the case of general joint distributions. We investigate the problem from an automated mechanism design perspective, meaning that we want to understand the algorithmic complexity of finding the optimal mechanism when we are given a finite set of type profiles and their distribution.

We show that the optimal deterministic, dominant strategy incentive compatible, ex-post individual rational mechanism can be computed in polynomial time by reducing the problem to finding a maximal weight closure in a directed graph. Node weights in the graph correspond to conditional virtual values. When valuations are independently distributed, the constructed mechanism is also optimal among all Bayes-Nash implementable and ex-interim individual rational mechanisms. In contrast, for dependent valuations strictly higher profit can be achieved if one allows for ex-interim individual rationality. By invoking techniques due to Crémer and McLean, we show that optimal deterministic, ex-interim individual rational, Bayes-Nash implementable or dominant strategy implementable mechanisms still can be found in polynomial time if the joint distribution of types satisfies certain regularity conditions.
\end{abstract}

JEL Codes: C61, C72, D82, H41

\section{Introduction}

We study the problem of finding a mechanism that maximizes the expected profit of a monopolistic seller of a single, non-excludable public good. This problem was first solved by Güth and Hellwig [1986] for the case of Bayesian-Nash implementation when valuations are independently distributed with monotone hazard rate. Our goal is to study the problem for general distributions of valuations, for dominant strategy and Bayes-Nash implementation, and for ex-post as well as

${ }^{*}$ Corresponding author 
interim individual rationality. It is too much to hope for closed form solutions in each case. Instead, the mechanism design problem is modeled as a combinatorial optimization problem, more precisely, as an integer linear program (ILP). The ILP has as set of feasible solutions the set of all mechanisms satisfying particular incentive and individual rationality constraints, and as objective the expected profit. The parameters of the constraints and the objective are determined by the set of possible type profiles and their distribution. One of the challenges is to represent the expected profit as a linear function in the decision variables, the other is to provide, if possible, an efficient algorithm to solve the integer linear program. We are interested in polynomial time algorithms in the number of agents and the number of type profiles of the agents. We are also interested in gaining insights in economic properties of optimal mechanisms, and classes of instances where a "simple" mechanism is optimal.

Our main result is that the profit maximizing, ex-post individual rational, dominant strategy incentive compatible mechanism can be computed in polynomial time. This holds for any number of agents, which is in sharp contrast to a recent result in Papadimitriou and Pierrakos [2011] on profit-maximizing single-item auctions for general distributions of valuations. Papadimitriou and Pierrakos [2011] reduce the optimal auction problem to finding the maximum weight independent set on a $k$-partite graph, where $k$ is the number of bidders. This yields a polynomial time algorithm for the two bidder case. They further show that the problem is NP-hard for more than two bidders. We show that finding a profit-maximizing mechanism for the non-excludable public good problem for general distributions can be modeled as an integer linear program with a totally unimodular matrix. More specifically, the ILP is equivalent to a maximal closure problem on partially ordered sets, where weights of the elements are equal to conditional virtual values. Contrary to single-item auctions, this yields a polynomial-time algorithm for any number of agents. Notably, the optimal mechanism might choose to provide the public good for type realizations where the sum of conditional virtual values is strictly smaller than the cost of providing the good. For the case of independent valuations with monotone hazard rate, we get the optimal Bayes-Nash allocation rule of Güth and Hellwig [1986] as a byproduct.

For general independent type distributions, we illustrate, using recent results by Gershkov et al. [2012], that allowing for interim individual rationality instead of only for ex-post individual rationality or Bayes-Nash implementation instead of only dominant strategy implementation cannot increase the expected profit. However, by virtue of an example it is shown that for correlated valuations Bayes-Nash implementation may yield more profit than dominant strategy implementation. Transferring techniques developed for auctions by Crémer and McLean [1988] to the public good case, we illustrate that under some mild assumptions the profit maximizing interim individual rational dominant strategy implementable mechanism can be determined in polynomial time. Furthermore as for auctions, it yields full surplus extraction. The same holds for Bayes-Nash implementation. By virtue of an example it is shown that full surplus extraction breaks down if we require ex-post individual rationality. It remains an open problem whether finding the optimal Bayes-Nash incentive compatible, ex-post individual rational mechanism can be efficiently computed, and how to compute efficiently the optimal Bayes-Nash incentive compatible, interim 
individual rational mechanism for general correlated valuations.

\section{$2 \quad$ Model and Preliminaries}

We are given a set of agents $N=\{1, \ldots, n\}$ who hold private information $t_{i} \in T_{i} \subseteq \mathbb{R}$ about their values for consumption of a non-excludable public good. We denote the set of type tuples of all agents by $T:=T_{1} \times \ldots \times T_{n}$. All sets $T_{i}$ are assumed to be finite. Type tuples $t \in T$ occur according to the joint probability distribution $\varphi$ and the cumulative distribution $\Phi$. Furthermore, let $\varphi_{t_{i}}$ be the probability that $t_{i}$ occurs and $\Phi_{t_{i}}$ the corresponding cumulative distribution function.

The set of possible outcomes is $A:=\{0,1\}$, where 1 denotes the event of providing the public good, while 0 means not providing it. Agents assess outcomes through valuation functions $v_{i}:=A \times T_{i} \rightarrow \mathbb{R}$ for all $i \in N$. In case of the non-excludable public good problem valuation functions take the form of $v_{i}\left(1, t_{i}\right)=t_{i}$ and $v_{i}\left(0, t_{i}\right)=0$. Agents act via a direct mechanism $(f, p)$, which consists of an allocation rule $f: T \rightarrow A$ and a payment scheme $p: T \rightarrow \mathbb{R}^{n}$ as we allow for transfers. Agents are assumed to have quasi-linear utilities implying that for type $t_{i}$ and reports $\left(s_{i}, t_{-i}\right) \in T$ the utility of agent $i$ is $v_{i}\left(f\left(t_{i}, t_{-i}\right), t_{i}\right)-p_{i}\left(s_{i}, t_{-i}\right)$.

The provision of the public good costs $C$. The profit $\pi(t)$ of the designer for a reported type tuple $t$ is the sum of payments collected minus the cost incurred:

$$
\pi(t)=\sum_{i=1}^{n} p_{i}(t)-f(t) C .
$$

We are seeking for mechanisms $(f, p)$ that maximize the expected profit $\sum_{t} \varphi_{t} \pi(t)$. First, we search for the optimal mechanism among those that are implementable in dominant-strategies and ex-post individually rational. Then we discuss other solution concepts. By the revelation principle we may restrict ourselves to direct mechanisms.

Definition 2.1 A mechanism $(f, p)$ is dominant strategy incentive compatible if and only if for all agents $i$, for all fixed $t_{-i}$ and for all $s_{i} \neq t_{i}$

$$
v_{i}\left(f\left(t_{i}, t_{-i}\right), t_{i}\right)-p_{i}\left(t_{i}, t_{-i}\right) \geq v_{i}\left(f\left(s_{i}, t_{-i}\right), t_{i}\right)-p_{i}\left(s_{i}, t_{-i}\right) .
$$

Definition 2.2 A mechanism $(f, p)$ is ex-post individual rational if and only if for all agents $i$ and for all $t \in T$

$$
v_{i}\left(f\left(t_{i}, t_{-i}\right), t_{i}\right)-p_{i}\left(t_{i}, t_{-i}\right) \geq 0 .
$$

Let $x_{t} \in\{0,1\}, t \in T$ be decision variables that represent the allocation function $f$, i.e., $x_{t}=1 \Leftrightarrow f(t)=1$. Let us also introduce decision variables for each payment, i.e., for all $i$ and $t$ we have that $p_{t}^{i}=p_{i}(t)$. Our mechanism design problem can then be formulated as the following 
integer linear program.

$$
\max \quad \sum_{t \in T} \varphi_{t}\left(\sum_{i} p_{t}^{i}-x_{t} C\right)
$$

subject to

$$
\begin{aligned}
t_{i} x_{t_{i}, t_{-i}}-p_{t_{i}, t_{-i}}^{i} & \geq t_{i} x_{s_{i}, t_{-i}}-p_{s_{i}, t_{-i}}^{i} & & \forall i \in N, \forall t_{-i} \in T_{-i}, \forall s_{i}, t_{i} \in T_{i}, \\
t_{i} x_{t}-p_{t}^{i} & \geq 0 & & \forall i \in N, t \in T, \\
x_{t} & \in\{0,1\} & & \forall t \in T
\end{aligned}
$$

Constraints (DS) ensure implementability in dominant strategies, while (IR) constraints are responsible for ex-post individual rationality. As we go for deterministic mechanisms we also require the allocation variables to be binary $(\mathrm{BI})$.

We refer to an allocation given by $x_{t_{i}, t_{-i}}$ as monotone allocation if and only if for all $i$ and $t_{-i}$, $x$ is increasing in $t_{i}$, i.e., for all $s_{i}, t_{i}$ if $s_{i}>t_{i}$, then $x_{s_{i}, t_{-i}} \geq x_{t_{i}, t_{-i}}$. It is a well-know result (see, e.g. Nisan et al. [2007]) that monotonicity in such single-parameter domains is necessary and sufficient for implementability in dominant strategies. Monotonicity implies that the allocation for each player given the others' types is a step-function that is based on critical values equal to the minimum type for which we allocate.

Proposition 2.3 Let $x$ be monotone. The maximal payments that can be charged in any dominant strategy and ex-post individual rational mechanism are given by:

$$
p_{t_{i}, t_{-i}}^{i}= \begin{cases}\inf \left\{s_{i} \in T_{i} \mid x_{s_{i}, t_{-i}}=1\right\} & \text { if } x_{t_{i}, t_{-i}}=1 \\ 0 & \text { if } x_{t_{i}, t_{-i}}=0\end{cases}
$$

This is a general result for single-parameter domains (see, for example, Nisan et al. [2007]). For the sake of completeness we include a proof in the appendix. Recall that our objective is to maximize the expected total profit, which is the total payment collected minus the cost of allocating the public good. Observe that in this case the payments should be maximal, hence, without loss of generality, we can specify the payment scheme for a profit maximizing implementable allocation $x$ as in (1).

Let us define the operator $\succ$ for vectors as follows: for every $s, t \in T$ we write $s \succ t$ if and only if $s_{i} \geq t_{i}$ for all $i \in N$ and $s \neq t$. Note that $\succ$ induces a partial order on $T$.

Proposition 2.4 Let $f$ be an allocation function and let $\left\{x_{t}\right\}_{t \in T}$ be the variables induced by $f$. Then $f$ is monotone if and only if for all $s, t \in T$ such that $s \succ t$ it holds that $x_{s} \geq x_{t}$.

Proposition 2.4 can be regarded as a special case of the PAD property (positive association of differences) that is implied by monotonicity (see Roberts [1979]). A simple proof can be found in the appendix. A set $S \subseteq T$ is called upper comprehensive if for all $s \in S, t \in T$, we have that $t \succ s$ implies $t \in S$. 
Corollary 2.5 An allocation function in the non-excludable public good setting is implementable if and only if the type tuples, for which we allocate the public good, form an upper comprehensive set on the partially ordered type space $(T, \succ)$.

\section{$3 \quad$ Finding the optimal mechanism}

In this section we provide an efficient algorithm to solve the integer linear program given by (P1),(DS), (IR), and (BI). First, we show how to eliminate variables $p_{t}^{i}$, second, we apply Corollary 2.5 to derive an integer linear program that can be solved in polynomial time. We conclude this section by discussing the special case of independent type distributions and relating our results to the results by Güth and Hellwig [1986].

\subsection{Linearization of the objective function}

Notice that the objective function is not linear in $x_{t}$, if we substitute variables $p$ as given in (1). In order to get a linear expression in $x$ we first reformulate the revenue part of the objective as

$$
\begin{aligned}
\sum_{t} \varphi_{t} \sum_{i} p_{t}^{i} & =\sum_{i} \sum_{t} \varphi_{t} p_{t}^{i} \\
& =\sum_{i} \sum_{t_{-i}} \sum_{t_{i}} \varphi_{t_{i}, t_{-i}} p_{t_{i}, t_{-i}}^{i} .
\end{aligned}
$$

Due to monotonicity, whenever for a fixed $t_{-i}$ there is some $t_{i}$ such that $x_{t_{i}, t_{-i}}=1$, then for all $s_{i} \geq t_{i}$ we have that $x_{s_{i}, t_{-i}}=1$, hence for that fixed $t_{-i}$ the sum of payments from agent $i$ is

$$
\sum_{t_{i}} \varphi_{t_{i}, t_{-i}} p_{t_{i}, t_{-i}}^{i}=t_{i}^{*} \sum_{s_{i} \geq t_{i}^{*}} \varphi_{s_{i}, t_{-i}}
$$

where $t_{i}^{*}=\inf \left\{s_{i} \mid f\left(s_{i}, t_{-i}\right)=1\right\}$.

For economy of notation let us introduce $t_{i}^{+}:=\inf \left\{s_{i} \in T_{i} \mid s_{i}>t_{i}\right\}$, where for the maximal type of agent $i$, we interpret $t_{i}^{+}$as a dummy type that takes value zero and occurs with probability zero. Building on the monotonicity property of the allocation function we can construct a series of coefficients for the induced variables such that the sum of the weighted variables results in the same revenue as in (3):

$$
\sum_{t_{i}} x_{t_{i}, t_{-i}}\left(t_{i} \sum_{s_{i} \geq t_{i}} \varphi_{s_{i}, t_{-i}}-t_{i}^{+} \sum_{s_{i} \geq t_{i}^{+}} \varphi_{s_{i}, t_{-i}}\right)=t_{i}^{*} \sum_{s_{i} \geq t_{i}^{*}} \varphi_{s_{i}, t_{-i}} .
$$


Note that if for a fixed $t_{-i}$ there is no $t_{i}$ such that $x_{t_{i}, t_{-i}}=1$, then the coefficients remain valid as all of the corresponding $x_{t_{i}, t_{-i}}$ variables are equal to zero. Therefore, (3) can be rewritten as

$$
\begin{aligned}
\sum_{t_{i}} \varphi_{t_{i}, t_{-i}} p_{t_{i}, t_{-i}}^{i} & =\sum_{t_{i}} x_{t_{i}, t_{-i}}\left(t_{i} \sum_{s_{i} \geq t_{i}} \varphi_{s_{i}, t_{-i}}-t_{i}^{+} \sum_{s_{i} \geq t_{i}^{+}} \varphi_{s_{i}, t_{-i}}\right) \\
& =\sum_{t_{i}} x_{t_{i}, t_{-i}}\left(t_{i} \varphi_{t_{i}, t_{-i}}-\left(t_{i}^{+}-t_{i}\right) \sum_{s_{i} \geq t_{i}^{+}} \varphi_{s_{i}, t_{-i}}\right) .
\end{aligned}
$$

The coefficient of each $x_{t_{i}, t_{-i}}$ can be interpreted as the added revenue from agent $i$ if we allocate the public good at reported type tuple $\left(t_{i}, t_{-i}\right)$, versus only at $\left(t_{i}^{+}, t_{-i}\right)$. Plugging this into (2) results in

$$
\begin{aligned}
\sum_{t} \varphi_{t} \sum_{i} p_{t}^{i} & =\sum_{i} \sum_{t_{-i}} \sum_{t_{i}} x_{t_{i}, t_{-i}}\left(t_{i} \varphi_{t_{i}, t_{-i}}-\left(t_{i}^{+}-t_{i}\right) \sum_{s_{i} \geq t_{i}^{+}} \varphi_{s_{i}, t_{-i}}\right) \\
& =\sum_{t} x_{t} \sum_{i}\left(t_{i} \varphi_{t}-\left(t_{i}^{+}-t_{i}\right) \sum_{s_{i} \geq t_{i}^{+}} \varphi_{s_{i}, t_{-i}}\right) .
\end{aligned}
$$

From now on we assume that $\varphi_{t}>0$ for all $t$. This assumption facilitates an economic interpretation of the coefficients without making the notation too messy. All results with respect to the algorithmic complexity of our problem hold without this assumption. With the assumption we can further transform as follows.

$$
\begin{aligned}
\sum_{t} \varphi_{t} \sum_{i} p_{t}^{i} & =\sum_{t} \varphi_{t} x_{t} \sum_{i}\left(t_{i}-\left(t_{i}^{+}-t_{i}\right) \frac{\sum_{s_{i} \geq t_{i}^{+}} \varphi_{s_{i}, t_{-i}}}{\varphi_{t}}\right) \\
& =\sum_{t} \varphi_{t} x_{t} \sum_{i}\left(t_{i}-\left(t_{i}^{+}-t_{i}\right) \sum_{r_{i}} \varphi_{r_{i}, t_{-i}} \frac{\sum_{s_{i} \geq t_{i}^{+}} \varphi_{s_{i} \mid t_{-i}}}{\varphi_{t}}\right) \\
& =\sum_{t} \varphi_{t} x_{t} \sum_{i}\left(t_{i}-\left(t_{i}^{+}-t_{i}\right)\left(\frac{1-\Phi_{t_{i} \mid t_{-i}}}{\varphi_{t_{i} \mid t_{-i}}}\right)\right)
\end{aligned}
$$

where $\varphi_{t_{i} \mid t_{-i}}$ is the conditional probability that $s_{i}$ occurs assuming that the others have $t_{-i}$ and $\Phi_{t_{i} \mid t_{-i}}$ is the corresponding conditional cumulative distribution function.

The objective function of (P1) can then be expressed as

$$
\sum_{t} \varphi_{t}\left(\sum_{i} p_{t}^{i}-x_{t} C\right)=\sum_{t} x_{t} \varphi_{t}\left(\sum_{i}\left(t_{i}-\left(t_{i}^{+}-t_{i}\right)\left(\frac{1-\Phi_{t_{i} \mid t_{-i}}}{\varphi_{t_{i} \mid t_{-i}}}\right)\right)-C\right) .
$$

Let

$$
\mu_{t_{i} \mid t_{-i}}=t_{i}-\left(t_{i}^{+}-t_{i}\right)\left(\frac{1-\Phi_{t_{i} \mid t_{-i}}}{\varphi_{t_{i} \mid t_{-i}}}\right) .
$$

We call $\mu_{t_{i} \mid t_{-i}}$ the conditional virtual valuation of agent $i$ with type $t_{i}$ when the rest of the agents 
have type $t_{-i}$. In case of independent type distributions this notion coincides with the virtual valuation introduced by Myerson [1981]. Notice that if we interpret $\mu_{t_{i} \mid t_{-i}}$ as interdependent valuations of the agents then the objective function

$$
\sum_{t} x_{t} \varphi_{t}\left(\sum_{i} \mu_{t_{i} \mid t_{-i}}-C\right)
$$

can be regarded as expected welfare-maximization with respect to conditional virtual values. However, from what follows it can be seen that the profit maximizing mechanism will not necessarily take the decision that maximizes virtual welfare for each realization $t$ of types. In other words, the profit-maximizing mechanism might set $x_{t}=1$ even if its coefficient in the objective is negative.

\subsection{Polynomial time algorithms}

As the objective function has become linear, by introducing

$$
b_{t}=\varphi_{t}\left(\sum_{i} \mu_{t_{i} \mid t_{-i}}-C\right)
$$

we can reformulate our initial model (P1) to the following integer linear program to find the expected profit maximizing public good mechanism. Thereby, we make also use of Corollary 2.5.

$$
\max \quad \sum_{t} b_{t} x_{t}
$$

subject to

$$
\begin{aligned}
x_{s}-x_{t} & \geq 0 & & \forall s, t \in T \text { s.t. } s \succ t, \\
x_{t} & \in\{0,1\} & \forall t \in T &
\end{aligned}
$$

Notice that if $b_{t} \geq 0$ for some $t$ implies that $b_{s} \geq 0$ for all $s \succ t$, then the solution is trivial: set $x_{t}=1$ if and only if $b_{t} \geq 0$. In the general case, the following observation provides us with a polynomial time algorithm.

Proposition 3.1 The incentive compatibility constraints of program (P2) form a totally unimodular matrix.

Proof The coefficient matrix of the incentive compatibility constraints can be seen as the nodearc incidence matrix of the redirected comparability graph of the partially ordered set $T$. The vertices are the type vectors and the mapping of the variables to the type vectors is surjective. There is a directed arc between each comparable vector directed from the larger vector to the smaller one. In the node-arc incidence matrix for each arc the start node is indicated by 1 , whilst the end node is indicated by -1 , just like in the coefficient matrix of the constraints. The incidence matrix of any directed graph is totally unimodular (Schrijver [2003]). 
Corollary 3.2 The linear program relaxation of (P2) yields an integer optimal solution, hence the problem can be solved in time polynomial in $|N|$ and $|T|$.

We can view (P2) also as a maximal closure problem, which is defined by Picard [1976] as follows.

Definition 3.3 (Maximal closure problem) Given a directed graph $G=(V, A)$ where $V$ is the set of nodes and $A$ the set of arcs, a closure of $G$ is defined as a subset of vertices $Y$ such that if a vertex belongs to $Y$ then all its successors belong also to $Y$. If each vertex $v_{i}$ is associated with a real number $c_{i}$, then a maximal closure $Y^{*}$ of $G$ is defined as a closure of maximal value (i.e. $\sum_{v_{i} \in Y^{*}} c_{i}$ is maximal).

Picard [1976] provides a combinatorial algorithm to find the optimal solution. It is done by reducing the problem to a network flow instance. First, he gives an equivalent quadratic formulation of the constraints. Then, it turns out that the Lagrangian dual of this construction is essentially the same task as finding the minimum cut of an associated network, which can be solved in polynomial time by maximizing the flow on the related network.

Maximal closure problems are well studied by virtue of their connection to a wide range of practical problems from open pit mining, record segmentation in large shared data bases to portfolio selection under contingency constraints. For more examples see Faaland et al. [1990] and Hochbaum and Chen [2000]. The latter papers also provide an overview and comparison of network based algorithms and heuristics used in practice to deal with maximum closure problems.

As a summary of our discussion we get the following theorem.

Theorem 3.4 Finding the profit-maximizing, deterministic, ex-post individual rational and dominant strategy implementable mechanism for the provision of a single, non-excludable public good can be done in time polynomial in the number of agents and the number of possible type tuples $(|N|,|T|)$.

Güth and Hellwig [1986] characterize the profit-maximizing Bayesian-Nash implementable mechanism for the case that types are distributed independently. The next subsection compares their result to ours.

\subsection{Independent type distributions}

Suppose, that types are independently distributed, i.e., for all $t \in T$ we have that $\varphi_{t}=\prod_{i} \varphi_{t_{i}}$. Readily, in this case for all $t_{-i}$

$$
\mu_{t_{i} \mid t_{-i}}=\mu_{t_{i}}=t_{i}-\left(t_{i}^{+}-t_{i}\right)\left(\frac{1-\Phi_{t_{i}}}{\varphi_{t_{i}}}\right)
$$


This implies that we can express the coefficients of the objective function in terms of virtual valuations in the Myerson sense for both settings:

$$
b_{t}=\varphi_{t}\left(\sum_{i} \mu_{t_{i}}-C\right) .
$$

Observe that $b_{t}$ is non-negative if and only if the sum of virtual valuations at $t$ covers the cost.

Moreover, assuming type distributions with monotone non-decreasing hazard rate $\frac{\varphi_{t_{i}}}{1-\Phi_{t_{i}}}$ and types where $t_{i}^{+}=t_{i}+1$, the virtual valuations along with the sum of virtual valuations become monotone non-decreasing in $t$. This implies that if there is a $t$ such that $b_{t} \geq 0$, then for all $s \geq t$ we have that $b_{s} \geq 0$. Hence, the optimal solution can be characterized as follows: set $x_{t}=1$ if and only if $\sum_{i} \mu_{t_{i}} \geq C$. This coincides with the findings of Güth and Hellwig [1986], which implies that Bayesian-Nash implementation leads to the same optimal mechanism in case of independent type distributions. We elaborate on this observation in further details in Section 4.1.

\section{Other solution concepts}

In Section 3 we showed how to find the optimal deterministic, ex-post individual rational, dominant strategy implementable mechanism. Corollary 3.2 tells us that randomized algorithms cannot do better than deterministic ones due to the TUM structure of the constraints, hence the first requirement does not have an impact on the expected profit. In this section we investigate what happens if we relax the remaining two requirements. First, we analyze Bayesian-Nash implementation as a less demanding alternative for dominant strategy implementation, then we focus on the implications of interim individual rationality.

Definition 4.1 A mechanism, represented by its induced variables $\left\{x_{t}\right\}_{t \in T}$ and $\left\{p_{t}^{i}\right\}_{i \in N, t \in T}$, is Bayesian-Nash implementable if and only if for all $i \in N$ and $t_{i}, s_{i} \in T_{i}$ we have that

$$
\sum_{t_{-i}} \varphi_{t_{-i} \mid t_{i}}\left(t_{i} x_{t_{i}, t_{-i}}-p_{t_{i}, t_{-i}}^{i}\right) \geq \sum_{t_{-i}} \varphi_{t_{-i} \mid t_{i}}\left(t_{i} x_{s_{i}, t_{-i}}-p_{s_{i}, t_{-i}}^{i}\right)
$$

Definition 4.2 A mechanism, represented by its induced variables $\left\{x_{t}\right\}_{t \in T}$ and $\left\{p_{t}^{i}\right\}_{i \in N, t \in T}$, is interim individual rational if and only if for all $i \in N$ and $t_{i} \in T_{i}$ we have that

$$
\sum_{t_{-i}} \varphi_{t_{-i} \mid t_{i}}\left(t_{i} x_{t_{i}, t_{-i}}-p_{t_{i}, t_{-i}}^{i}\right) \geq 0
$$

\subsection{Bayesian-Nash implementation}

Recent papers provide conditions under which Bayesian-Nash and dominant strategy implementation are equivalent for one-dimensional types that are distributed independently, and valuations for outcomes that are linear functions of agents' types. Gershkov et al. [2011] show that in case of two outcomes for any Bayesian incentive compatible mechanism there is a dominant-strategy incentive compatible mechanism that generates the same interim probabilities with which each 
alternative is selected. The paper provides an example for a three outcome case where the equivalence brakes down. A positive result for environments with more than two outcomes is given in Manelli and Vincent [2010], who prove the equivalence for not necessarily symmetric, singleitem auction settings. If we define equivalence in a weaker sense, i.e, two implementations are equivalent if they generate the same interim utilities for each agent, then according to Gershkov et al. [2012] Bayesian-Nash and dominant strategy implementation remains equivalent in general in environments with more than two outcomes. All these results require that the type space is single-dimensional.

Corollary 4.3 In the profit-maximizing non-excludable public good problem where the types are independently distributed we can restrict ourselves to dominant strategy implementation without loss of generality.

Proof Observe that this problem is a special case of the setting discussed in Gershkov et al. [2011] which implies that for every Bayesian incentive compatible mechanism there is a dominant strategy incentive compatible one that provides the same interim probabilities of providing the public good. In this case we can construct transfers for the dominant strategy implementable mechanism using the payments of the Bayesian mechanism in such a way that the expected revenues of the two mechanisms are equal (see Gershkov et al. [2012]).

This result does not extend to the case of correlated type distributions as we can see from the following example.

Example 4.4 Let $N=\{1,2\}, T_{1}=T_{2}=\{1,2\}, C=1$ and $\varphi_{1,1}=0.4, \varphi_{1,2}=0.1, \varphi_{2,1}=0.1$, $\varphi_{2,2}=0.4$. Solving this problem with our approach yields the optimal solution given in the following table.

\begin{tabular}{c|c|c|c}
$t$ & $x_{t}$ & $p_{t}^{1}$ & $p_{t}^{2}$ \\
\hline$(1,1)$ & 0 & 0 & 0 \\
$(1,2)$ & 0 & 0 & 0 \\
$(2,1)$ & 0 & 0 & 0 \\
$(2,2)$ & 1 & 2 & 2
\end{tabular}

This mechanism yields an expected profit of 1.2, which is by definition the highest expected revenue that any ex-post individual rational and dominant strategy implementable mechanism can attain in this setting. Next, consider the following mechanism.

\begin{tabular}{c|c|c|c}
$t$ & $x_{t}$ & $p_{t}^{1}$ & $p_{t}^{2}$ \\
\hline$(1,1)$ & 1 & 1 & 1 \\
$(1,2)$ & 0 & 0 & 0 \\
$(2,1)$ & 0 & 0 & 0 \\
$(2,2)$ & 1 & 1.75 & 1.75
\end{tabular}

Notice that the allocation rule of this mechanism is not monotone, hence the mechanism is not dominant strategy incentive compatible. Observe, on the other hand, that it is Bayesian incentive compatible and its expected profit is 1.4 . 
Thus, in case of correlated type distributions there can be Bayesian-Nash implementable mechanisms for the non-excludable public good problem that generate higher revenue than any mechanism implementable in dominant strategies.

\subsection{Interim individual rationality}

Interim individual rationality constraints can be modeled as Bayesian incentive compatibility constraints with dummy types and the same can be done with ex-post individual rationality and dominant strategy incentive compatibility constraints (see Vohra [2011] for further details). Corollary 4.3 tells us that the optimal mechanisms yield the same revenue for both notions of implementation when types are distributed independently. Hence we can conclude the following.

Corollary 4.5 In the profit-maximizing, non-excludable public good problem where the types are independently distributed we can restrict ourselves to interim individual rationality without loss of generality.

Example shows that in case of correlated types the equivalence of Bayesian-Nash and dominant strategy implementation does not hold, hence we can as well expect that interim individual rationality provides us more flexibility to elicit payments. An key result concerning the limitations of ex-post individual rationality compared to interim individual rationality is due to Crémer and McLean [1988]. They deal with single-item auction settings. Given a fixed type space, they provides conditions on the joint distribution of these types that allow full extraction of the agents' surplus by interim individual rational mechanisms which are either Bayesian-Nash or dominant strategy implementable. When these conditions are met, full surplus extraction can be achieved by a Vickrey auction with modified payments. To explain the conditions in our context, consider an agent $i$ and let $\Gamma^{i}$ denote the $T_{i} \times T_{-i}$ matrix whose elements are the conditional probabilities of the joint distribution function, that is, $\Gamma_{t_{i}, t_{-i}}^{i}=\varphi_{t_{-i} \mid t_{i}}$. For dominant strategy implementation the condition is that for each agent $i$ the columns of $\Gamma^{i}$ have to be linearly independent. For Bayesian implementation the condition is milder: it requires only the conic independence of the columns of $\Gamma^{i}$ (i.e. no column is contained in the cone spanned by the other columns). For a mechanism given by its induced variables $\left\{x_{t}\right\}_{t \in T}$ and $\left\{p_{t}^{i}\right\}_{t \in T, i \in N}$, let us define the expected social surplus as

$\sum_{t} \varphi_{t} x_{t}\left(\sum_{i} t_{i}-C\right)$. The expected profit of the public good provider is always less than or equal to the expected social surplus due to (5). It is a folklore result that the expected social surplus is maximized by mechanisms that allocate if and only if the sum of valuations exceed the cost (see, for example, Nisan et al. [2007]). Therefore the expected surplus arising from such a mechanism is an upper bound on the maximal expected profit that can be achieved for a given instance. If a mechanism yields profit equal to this upper bound, we say that the mechanism extracts the full social surplus.

Theorem 4.6 Take an instance of the profit-maximizing, non-excludable public good problem. Assume that the type distribution satisfies the following: for each $i \in N$ there does not exist a 
vector of $\left\{\lambda_{t_{i}}\right\}_{t_{i} \in T_{i}}$, not all equal to zero, such that

$$
\sum_{t_{i} \in T_{i}} \lambda_{t_{i}} \varphi_{t_{-i} \mid t_{i}}=0 \quad \forall t_{-i} \in T_{-i}
$$

Then the profit-maximizing, dominant strategy implementable and interim individual rational mechanism can be constructed in time polynomial in $|T|$ and $|N|$. Moreover, the optimal mechanism is deterministic and extracts the full social surplus.

Theorem 4.7 Take an instance of the profit-maximizing, non-excludable public good problem. Assume that the type distribution satisfies the following: for each $i \in N$ and each $t_{i} \in T_{i}$ there does not exist a vector $\left\{\lambda_{s_{i}}\right\}_{s_{i} \in T_{i} \backslash t_{i}}$ such that

$$
\lambda_{s_{i}} \geq 0 \quad \forall s_{i} \in T_{i} \backslash t_{i}
$$

and

$$
\varphi_{t_{-i} \mid t_{i}}=\sum_{s_{i} \in T_{i} \backslash t_{i}} \lambda_{s_{i}} \varphi_{t_{-i} \mid s_{i}} \quad \forall t_{-i} \in T_{-i}
$$

Then the profit-maximizing, Bayesian-Nash implementable and interim individual rational mechanism can be constructed in time polynomial in $|T|$ and $|N|$. Moreover, the optimal mechanism is deterministic extracts the full social surplus.

The proofs for both Theorems are based on mild modifications of the construction given in Crémer and McLean [1988] and the observation that these constructions can be done in polynomial time. We provide them in the appendix.

We emphasize that determining whether a distribution satisfies the conditions of either Theorem 4.6 or Theorem 4.7 can be done in polynomial time. The linear independence of the conditional probability matrices can be done by Gaussian elimination, whilst the question of conic independence can be reduced to a feasibility check of a series of linear programs (one program per column).

In terms of surplus extraction, the assumptions in Theorem 4.6 and Theorem 4.7 are not just sufficient, but also necessary in the following sense. Whenever a joint type distribution fails condition (6) or (7) and (8) we can construct a $T$ such that full surplus extraction is not possible in the corresponding settings (a proof can be given by modifying the proof for auctions by Crémer and McLean [1988], but is omitted here). Independent distributions violate the assumptions of both theorems, which explains partly why we cannot achieve more revenue with interim individual rationality than with ex-post individual rationality under such distributions.

We conclude this section with an example to show that the equivalence of ex-post and interim individual rationality is no longer valid under correlated types.

Example 4.8 Consider the following mechanism for the setting in Example 4.2. 


\begin{tabular}{c|c|c|c}
$t$ & $x_{t}$ & $p_{t}^{1}$ & $p_{t}^{2}$ \\
\hline$(1,1)$ & 1 & $2 / 3$ & $2 / 3$ \\
$(1,2)$ & 1 & $2 \frac{1}{3}$ & $2 / 3$ \\
$(2,1)$ & 1 & $2 / 3$ & $2 \frac{1}{3}$ \\
$(2,2)$ & 1 & $2 \frac{1}{3}$ & $2 \frac{1}{3}$
\end{tabular}

Observe that the proposed mechanism is not ex-post individual rational, but dominant strategy incentive compatible, interim individual rational and yields an expected profit of 2. Therefore it extracts the whole social surplus, which is not possible when we require ex-post individual rationality. (Indeed, the second mechanism from Example 4.2 has been verified by a computer program to be optimal.)

\section{Conclusions}

We have studied the problem of finding the optimal, deterministic, mechanism for providing a non-excludable public good when valuations are correlated. We considered dominant strategy as well as Bayesian Nash implementation, and ex-post as well as ex-interim individual rational mechanism. The results and open questions can be summarized in the following overview of requirements:

1. The optimal dominant strategy incentive compatible, ex-post individual rational mechanism can be computed in polynomial time.

2. The optimal dominant strategy incentive compatible, ex-interim individual rational mechanism can be computed in polynomial time if the conditions of Theorem 4.6 hold. Example 4.8 shows that it may yield strictly higher revenue than the optimal mechanism for requirements as in (1). It remains an open problem how to efficiently compute the optimal deterministic mechanism for general dependent distributions.

3. The computation of the optimal Bayes-Nash incentive compatible, ex-interim individual rational mechanism remains an open problem. Example 4.2 shows that it may yield strictly higher revenue than the optimal mechanism for requirements as in (1).

4. The optimal Bayesian Nash incentive compatible, ex-interim individual rational mechanism can be computed in polynomial time if the conditions of Theorem 4.7 hold. It follows from Crémer and McLean [1988] that the optimal mechanism can yield strictly higher profit than the optimal mechanism for requirements as in (2). It remains an open problem how to efficiently compute the optimal deterministic mechanism for general dependent distributions.

In each of the cases where we are able to show polynomial time solvability, randomized mechanisms cannot yield more revenue than deterministic mechanisms. 


\section{References}

Edward Clarke. Multipart pricing of public goods. Public Choice, 11(1):17-33, 1971. ISSN 0048-5829. doi: 10.1007/BF01726210.

Jacques Crémer and Richard P. McLean. Full extraction of the surplus in Bayesian and dominant strategy auctions. Econometrica, 56(6):1247-1257, 1988. ISSN 00129682. doi: 10.2307/1913096.

Bruce Faaland, Kiseog Kim, and Tom Schmitt. A new algorithm for computing the maximal closure of a graph. Management Science, 36(3):315-331, 1990.

Alex Gershkov, Benny Moldovanu, and Xianwen Shi. Bayesian and dominant strategy implementation revisited. Working Papers tecipa-422, University of Toronto, Department of Economics, February 2011. URL http://ideas.repec.org/p/tor/tecipa/tecipa-422.html.

Alex Gershkov, Jacob K. Goeree, Alexey I. Kushnir, Benny Moldovanu, and Xianwen Shi. On the equivalence of Bayesian and dominant strategy implementation. Econometrica, forthcoming, 2012 .

Werner Güth and Martin Hellwig. The private supply of a public good. Journal of Economics, 5:121-159, 1986. doi: 10.1007/BF03051789. URL http://dx.doi.org/10.1007/BF03051789.

Dorit S. Hochbaum and Anna Chen. Performance analysis and best implementations of old and new algorithms for the open-pit mining problem. Operations Research, 48(6):pp. 894-914, 2000. ISSN 0030364X. URL http://www.jstor.org/stable/222997.

Alejandro Manelli and Daniel Vincent. Bayesian and dominant-strategy implementation in the independent private-values model. Econometrica, 78(6):1905-1938, 2010. doi: 10.3982/ ECTA8025.

Roger Myerson. Optimal auction design. Mathematics of Operations Research, 6(1):58-73, 1981.

Noam Nisan, Tim Roughgarden, Eva Tardos, and Vijay V. Vazirani. Algorithmic Game Theory. Cambridge University Press, New York, NY, USA, 2007. ISBN 0521872820.

Christos H. Papadimitriou and George Pierrakos. On optimal single-item auctions. In Proceedings of the 43rd annual ACM symposium on Theory of computing, STOC '11, pages 119-128, New York, NY, USA, 2011. ACM. ISBN 978-1-4503-0691-1. doi: http://doi.acm.org/10.1145/ 1993636.1993654. URL http://doi.acm.org/10.1145/1993636.1993654.

Jean-Claude Picard. Maximal closure of a graph and applications to combinatorial problems. Management Science, 22(11):pp. 1268-1272, 1976. ISSN 00251909. URL http://www.jstor . org/stable/2630227.

Kevin Roberts. The characterization of implementable choice rules. In Jean-Jacques Laffont, editor, Aggregation and Revelation of Preferences, pages 321-349. North-Holland, 1979. URL http://www.nuffield.ox.ac.uk/users/robertsk/Roberts(1979).pdf. 
Alexander Schrijver. Combinatorial optimization. Springer, 2003. ISBN 9783540443896. URL http://books . google.com/books?id=mqGeSQ6dJycC.

R.V. Vohra. Mechanism Design: A Linear Programming Approach. Econometric Society Monographs. Cambridge University Press, 2011. ISBN 9780521179461. URL http://books .google. nl/books?id=YSzVz81hjycC.

\section{Appendix}

Proof of Proposition 2.3. We leave it to the reader to verify that the payments given in (1) provide ex post individual rational, dominant strategy incentive compatible payments for the allocation rule given by $x$.

Now let $p$ be any incentive compatible, ex-post individual rational payments for $x$. Observe that if $x_{t_{i}, t_{-i}}=x_{s_{i}, t_{-i}}$, then changing the role of $s_{i}$ and $t_{i}$ in inequality (DS) implies that $p_{t_{i}, t_{-i}}^{i}=$ $p_{s_{i}, t_{-i}}^{i}$. This is called taxation principle.

Now let $x_{t_{i}, t_{-i}}=1$. By (IR) we get $p_{t_{i}, t_{-i}} \leq t_{i}$. In combination with the observation this yields

$$
p_{t_{i}, t_{-i}} \leq \inf \left\{s_{i} \mid f\left(s_{i}, s_{-i}\right)=1\right\}
$$

Finally, let $x_{t_{i}, t_{-i}}=0$. By (IR) we get $p_{t_{i}, t_{-i}} \leq 0$.

Proof of Proposition 2.4. The "if" part follows from the fact that the theorem is a generalization of the monotonicity constraints.

To prove the "only if" part, take any $t, s \in T$ such that $s \succ t$. Let $I$ be the index set for dimensions $i$ where $s_{i}>t_{i}$. Let us assume, without loss of generality, that $s$ and $t$ differs in the first $m$ dimensions, so $I=\{1, \ldots, m\}$. Consider the series of types $\left(t^{0}, \ldots, t^{m}\right)$, where $t^{i}=\left(s_{1}, s_{2}, \ldots, s_{i}, t_{i+1}, \ldots, t_{m}, \ldots\right)$. Clearly, $t^{0}=t$ and $t^{m}=s$. For all $i$ by assumption and

by the construction it holds that $t_{j}^{i}=t_{j}^{i-1}$ for $j \neq i$ and $t_{i}^{i}>t_{i}^{i-1}$. Monotonicity implies that $x_{t^{i}} \geq x_{t^{i-1}}$, hence

$$
x_{s}=x_{t^{m}} \geq x_{t^{n-1}} \geq \ldots \geq x_{t^{0}}=x_{t} .
$$

Proof of Theorem 4.6. The efficient allocation rule given by $x_{t}=1$ if and only if $\sum_{i \in N} t_{i} \geq C$, together with payments $p_{t}^{i}$ set according to (1) provide the pivotal mechanism introduced by Clarke [1971]. We show how to modify the payments $p_{t_{i}, t_{-i}}^{i}$ by adding terms $g_{t_{-i}}^{i}$ such that the resulting mechanism is interim individual rational and extracts in expectation full surplus. Such a modification does not harm dominant strategy incentive compatibility as the terms do not depend on $t_{i}$.

Introduce for all $i \in N$ and $t_{i} \in T_{i}$

$$
h_{t_{i}}^{i}=\sum_{t_{-i}} \varphi_{t_{-i} \mid t_{i}}\left(t_{i} x_{t_{i}, t_{-i}}-p_{t_{i}, t_{-i}}^{i}\right)
$$


to measure the expected utility of agent $i$ when he possesses type $t_{i}$. Condition (6) ensures that $\Gamma^{i}$ has full row rank, hence there exist vectors $\left(g_{t_{-i}}^{i}\right)_{t_{-i} \in T_{-i}}$ such that for all $t_{i} \in T_{i}$ we have that

$$
\sum_{t_{-i} \in T_{-i}} \varphi_{t_{-i} \mid t_{i}} g_{t_{-i}}^{i}=h_{t_{i}}^{i}
$$

Now modify payments $p$ as follows: $\hat{p}_{t_{i}, t_{-i}}^{i}=p_{t_{i}, t_{-i}}^{i}+g_{t_{-i}}^{i}$. By definition

$$
\sum_{t_{-i}} \varphi_{t_{-i} \mid t_{i}}\left(t_{i} x_{t_{i}, t_{-i}}-\hat{p}_{t_{i}, t_{-i}}^{i}\right)=0
$$

that is, expected utility of each agent is 0 . Thus the expected profit of the mechanism designer equals social surplus. By that it attains the upper bound on the maximal profit and the mechanism is optimal. Observe that the pivotal allocation function can be constructed in constant time and the payment can be determined also in polynomial time using Gaussian elimination for instance.

Proof of Theorem 4.7 As in the proof of Theorem 4.6 we choose the allocation function that maximizes social surplus: $x_{t}=1$ if and only if $\sum_{i \in N} t_{i} \geq C$.

By the assumptions that none of the rows of the matrices $\Gamma^{i}$ is contained in the cone spanned by the other rows of the same matrix, we get from Farka's Lemma that there exist for each $i \in N$ and $t_{i} \in T_{i}$ a vector $\left(g_{t_{i}}^{i}\right)_{t_{-i} \in T_{-i}}$ such that:

$$
\sum_{t_{-i} \in T_{-i}} \varphi_{t_{-i} \mid t_{i}} g_{t_{i}, t_{-i}}^{i}<0
$$

and

$$
\sum_{t_{-i} \in T_{-i}} \varphi_{t_{-i} \mid s_{i}} g_{t_{i}, t_{-i}}^{i} \geq 0 \quad \forall s_{i} \neq t_{i}
$$

Let $h_{t}^{i}=g_{t}^{i}-\sum_{t_{-i} \in T_{-i}} \varphi_{t_{-i} \mid t_{i}} g_{t_{i}, t_{-i}}^{i}$ for all $t \in T$. Then we have that

$$
\sum_{t_{-i} \in T_{-i}} \varphi_{t_{-i} \mid t_{i}} h_{t_{i}, t_{-i}}^{i}=0
$$

and

$$
\sum_{t_{-i} \in T_{-i}} \varphi_{t_{-i} \mid s_{i}} h_{t_{i}, t_{-i}}^{i}>0 \quad \forall s_{i} \neq t_{i}
$$

Now define payments as

$$
p_{t}^{i}=\sum_{t_{-i}} \varphi_{t_{-i} \mid t_{i}} t_{i} x_{t_{i}, t_{-i}}+M h_{t}^{i}
$$

for some large enough $M$. The construction of $h_{t}^{i}$ implies that $\sum_{t_{-i}} \varphi_{t_{-i} \mid t_{i}}\left(t_{i} x_{s_{i}, t_{-i}}-p_{s_{i}, t_{-i}}^{i}\right)=0$ if $s_{i}=t_{i}$ otherwise it is arbitrary negative, which ensures that the interim individual rationality constraints (5) are binding and the Bayesian incentive compatibility constraints (4) are satisfied. We get also that the mechanism extracts full social surplus, hence it is optimal. 
We next discuss the running time of constructing the optimal mechanism. The allocation function is given, thus it takes constant time to determine it. To calculate the payments, first, we have to find the collection $\left\{g_{t}^{i}\right\}_{t \in T}$, then set an appropriate $M$. The latter can be done in linear time, e.g., take

$$
M=\max _{i, t_{i}}\left\{\sum_{t_{-i}} \varphi_{t_{-i} \mid t_{i}} t_{i} x_{t_{i}, t_{-i}}\right\} / \min _{i, t_{i}}\left\{\sum_{t_{-i} \in T_{-i}} \varphi_{t_{-i} \mid t_{i}} h_{t_{-i}}^{i}\right\}
$$

For finding appropriate $\left\{g_{t}^{i}\right\}_{t \in T}$ for each agent $i$ and each $t_{i} \in T$ in polynomial time one can use the following linear program.

$$
\min \sum_{t_{-i} \in T_{-i}} \varphi_{t_{-i} \mid t_{i}} g_{t_{i}, t_{-i}}^{i}
$$

subject to

$$
\sum_{t_{-i} \in T_{-i}} \varphi_{t_{-i} \mid s_{i}} g_{t_{i}, t_{-i}}^{i} \geq 0 \quad \forall s_{i} \neq t_{i} .
$$

This completes the proof. 